IgG mediated effects via $\mathrm{AT}(1) \mathrm{R}$ and $\mathrm{ET}(\mathrm{A}) \mathrm{R}$ on different cytokines, growth factors and cell viability were measured by for example, toxicity test, qRT-PCR and ELISA.

Results Treatment of HMEC-1 cells with SSc- IgG led to a strong upregulation of several mediators compared to negative control treatment. In case of IL-8, the mRNA and protein expression levels were upregulated. Moreover, expression of mRNA was downregulated and partially reduced on the protein levels using pre- treatment with receptor inhibitors. Interestingly, treatment with natural ligands did not result in interleukin 8 (IL-8) up-regulation. Treatment with SSc-IgG led also to a significantly reduced cell viability compared to negative control treatment. These effects were partially abolished by pretreatment with AT(1)R- inhibitor, but completely abolished using one but not another ET - inhibitor.

Conclusion Our results suggest an autoantibody-driven cytotoxicity and inflammatory activation of endothelial cells by angiotensin/endothelin-receptors in vitro. The data also suggest a high heterogeneity of antibody-mediated effects within IgG samples from different SSc patients, a role of other possible autoantibodies present in the SSc-IgG, and different responses to inhibitors and natural ligands. Whether these in vitro data could be used to identify responders or non- responsers to therapy remains to be studied. In vivo experiments are underway to give better insight into the complex nature of the SSc-antibody-mediated effects.

\title{
17 ANTI-AT(1)R AND ANTI-ET(A)R AUTOANTIBODIES IN SYSTEMIC SCLEROSIS: CLUES FOR POSSIBLE INVOLVEMENT IN DISEASE PATHOLOGY
}

Angela Kill, Mike 0 Becker, Jeannine Guenther, Harald Heidecke, Duska Dragun and Gabriela Riemekaste Charité University Hospital and German Rheumatology Research Center, a Leibniz Institute, Berlin, Germany

\subsection{6/annrheumdis-2011-201234.17}

Background Autoantibodies that target the angiotensin-II type-1 receptor (AT1R-Abs) and the endothelin-1 receptor type A (ETAR-Abs) were recently identified in systemic sclerosis (SSc). They showed strong association with clinical symptoms such as vascular and fibrotic complications including development of pulmonary arterial hypertension (PAH) and death, thus implicating their contribution to SSc pathogenesis. Here, different autoantibody-mediated effects and their blockade by receptor inhibitors were studied in an in vitro setting.

Methods Human microdermal endothelial cells-1 (HMEC-1) were treated with IgG from SSc patients containing anti-AT(1) $\mathrm{R}$ and anti-ET(A)R autoantibodies and with IgG of healthy donors as a negative control. In parallel, cells were pre- treated with various receptor antagonists alone and in combination. 